40th "Jaszowiec" International School and Conference on the Physics of Semiconductors, Krynica-Zdrój 2011

\title{
Coupling between Electronic and Vibrational Excitations in Carbon Nanotubes Filled with $\mathrm{C}_{60}$ Fullerenes
}

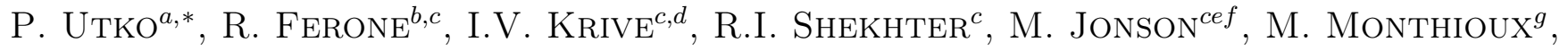 \\ L. NOÉ ${ }^{g}$ AND J. NYGARD ${ }^{a}$ \\ ${ }^{a}$ Nano-Science Center \& Niels Bohr Institute, University of Copenhagen, DK-2100 Copenhagen, Denmark \\ ${ }^{b}$ Department of Physics, Lancaster University, Lancaster LA1 4YB, UK \\ ${ }^{c}$ Department of Physics, University of Gothenburg, SE-412 96 Göteborg, Sweden \\ ${ }^{d}$ B.I. Verkin Institute for Low Temperature Physics and Engineering, 61103 Kharkov, Ukraine \\ ${ }^{e}$ School of Engineering and Physical Sciences, Heriot-Watt University, Edinburgh EH14 4AS, UK \\ ${ }^{f}$ School of Physics, Konkuk University, Seoul 143-701, Korea \\ ${ }^{g}$ CEMES-CNRS, B.P. 94347, F-31055 Toulouse Cedex 4, France
}

\begin{abstract}
We investigate the low-temperature electron transport through $\mathrm{C}_{60}$ peapods, which are carbon nanotubes encapsulating $\mathrm{C}_{60}$ fullerenes. We find that the temperature dependence of the Coulomb blockade oscillations in peapod quantum dots deviates from conventional Breit-Wigner behavior, showing signatures of the Franck-Condon blockade of conductance. This indicates the presence of a coupling between electronic states and vibrational excitations. Unlike for suspended empty nanotubes, these are not intrinsic vibrational modes of the tube but mechanical vibrations of the encapsulated fullerenes that affect the electronic transport. Fullerene peapods thus emerge as a new class of nanoelectromechanical systems.
\end{abstract}

PACS: 73.63.Fg, 73.63.Kv, 73.23.Hk, 73.22.Lp, 71.38.--k

\section{Introduction}

Filling single wall carbon nanotubes with $\mathrm{C}_{60}$ fullerenes yields hybrid structures $[1,2]$, the so-called $\mathrm{C}_{60}$ fullerene peapods, whose potential nanoelectronic applications range from data storage devices [3] to spin-qubit arrays for quantum computing [4]. However, the effect of the entrapped fullerenes on the electronic properties of peapods still remains unclear. Theoretical investigations predict pronounced changes in the electronic band structure of the nanotube [5], which is supported by the scanning tunneling microscopy [6] and photoluminescence [7] experiments. On the other hand, no fundamental perturbation in the electron system has been found in the photoemission [8] and electron transport [9] studies on peapods.

Here, we investigate the effect of $\mathrm{C}_{60}$ fullerenes on the low-temperature electron transport through peapod quantum dots operated in the regime of weak electronic coupling $\Gamma$ between energy states of the dot and its electrical leads [10]. We find that the temperature dependence of the Coulomb blockade (CB) oscillations differs from that for empty tubes, indicating the presence of a nanoelectromechanical coupling between electronic states of the nanotube and mechanical vibrations of fullerenes. This provides a useful method to detect the $\mathrm{C}_{60}$ presence and to probe the interplay between electrical and

* corresponding author; e-mail: pawel@fys.ku.dk mechanical excitations in peapods, which thus emerge as a new class of nanoelectromechanical systems. We note that other types of devices for which a coupling to mechanical vibrations plays a crucial role in electrical transport are suspended nanotubes [11], as well as molecular conductors and transistors.

\section{Experimental and results}

To enable electrical measurements, $\mathrm{C}_{60}$ peapods were deposited onto a $\mathrm{Si} / \mathrm{SiO}_{2}$ substrate. Individual peapods with a diameter of $1.3-2 \mathrm{~nm}$ were then located by atomic force microscopy and contacted with $\mathrm{Pd} / \mathrm{Au}$ electrodes using e-beam lithography. See Ref. [10] for more details on devices. A ${ }^{3} \mathrm{He}$ refrigerator with a base temperature of $T=300 \mathrm{mK}$ was used for measurements. At low temperatures, peapods were dominated by single-electron charging (see CB oscillations in Fig. 1a), with the charging energy $U \approx 8-12 \mathrm{meV}$ and the electronic level spacing $\Delta E \approx 2-3 \mathrm{meV}$. These energy scales corresponded well to a $400 \mathrm{~nm}$ wide gap between the source and drain electrodes, suggesting the formation of single peapod quantum dots.

For a quantum dot, the lineshape of the Coulomb blockade peaks is commonly described by the BreitWigner (BW) formula [12]:

$$
G(E)=\int\left(-\frac{\partial f\left(E^{\prime}\right)}{\partial E^{\prime}}\right) G_{\mathrm{BW}}\left(E-E^{\prime}\right) \mathrm{d} E^{\prime} .
$$

At low temperatures $k_{\mathrm{B}} T \ll \Gamma$, the CB peak approaches 

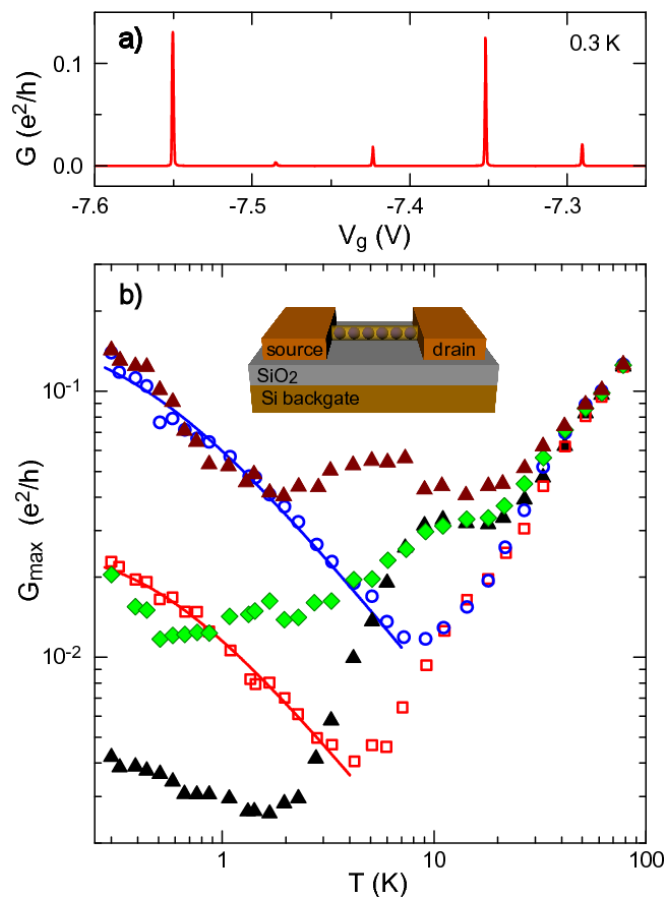

Fig. 1. (a) Linear conductance $G=\mathrm{d} I /\left.\mathrm{d} V\right|_{\mathrm{V}=0}$ as a function of gate voltage $V_{\mathrm{g}}$, measured for a $\mathrm{C}_{60}$ fullerene peapod at $0.3 \mathrm{~K}$. (b) Conductance amplitude $G_{\max }$ vs. temperature $T$, determined for the five Coulomb blockade peaks shown in (a). Peaks indicated with open symbols behave as predicted by the conventional BreitWigner model (the solid lines are fits to BW model), while the others show an abnormal temperature dependence. The inset shows a schematic layout of a peapod device.

the Lorentzian form of $G_{\mathrm{BW}}(E) \propto 1 /\left[\left(E-E_{0}\right)^{2}+(\Gamma / 2)^{2}\right]$. Whereas at higher temperatures $k_{\mathrm{B}} T \gg \Gamma$, it can be approximated by a negative derivative of the Fermi function $G(E) \propto-\partial f / \partial E$. Correspondingly, the maximal peak conductance $G_{\max }$ is nearly independent of temperature at $k_{\mathrm{B}} T \ll \Gamma$, and shows the $1 / T$ dependence at $k_{\mathrm{B}} T \gg \Gamma$.

However, unlike for empty nanotubes, $G_{\max }(T)$ for fullerene peapods often deviates from the Breit-Wigner model, see Fig. 1b. The anomaly typically occurs around few kelvin, i.e. at an energy scale $(\approx 0.1 \mathrm{meV})$ much smaller than that for electronic excitations $(\approx 1 \mathrm{meV})$ but relevant for fullerene vibrations within a longitudinal harmonic potential inside the nanotube. We note here that vibrations in transverse direction would have higher characteristic energies $(\approx 1 \mathrm{meV})$ as compared to the longitudinal ones, due to the low diameter of our peapods.

We therefore model the peapod as a multilevel quantum dot for which each quantized electronic level is selectively coupled to a single vibrational mode, as in the local Holstein model [13]. If the resonant tunneling electrons spend sufficiently long time in the dot, vibrational excitations lead to the formation of real electron-vibron hybrids - the Holstein polarons. Since the electron dwell time in the quantum dot scales as $\tau \propto 1 / \Gamma$, the polaronic effects become more apparent for weak electronic coupling $\Gamma$. See Refs. $[10]$ and [14] for more details on the model.

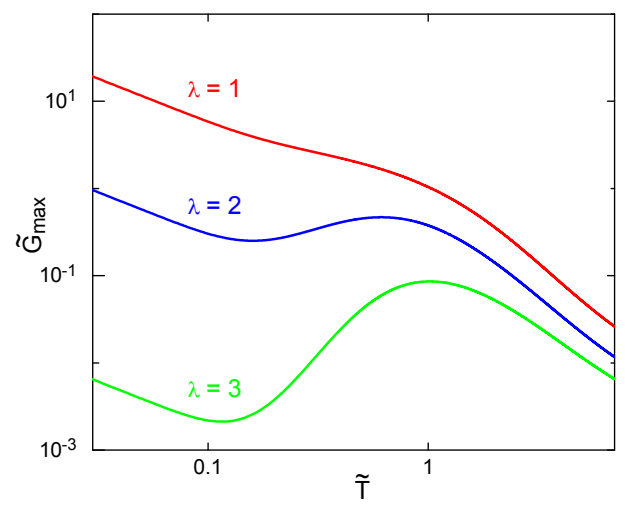

Fig. 2. Behavior of the renormalized peak conductance $\widetilde{G}_{\max }=G_{\max } \hbar \omega_{0} / \gamma$ vs. reduced temperature $\widetilde{T}=$ $k_{\mathrm{B}} T / \hbar \omega_{0}$ calculated for three values of the electronvibron coupling $\lambda$ using the polaronic model described in the main text.

Our model simulations show good correspondence to the experimental traces deviating from the Breit-Wigner behavior, see Fig. 2. At low temperatures $k_{\mathrm{B}} T \ll \hbar \omega_{0}$, the conductance becomes suppressed for strong electronvibron coupling $\lambda \gtrsim 1$, where $\hbar \omega_{0}$ is the vibrational quantum. Such a polaronic (Franck-Condon) blockade of the conductance [15] results from the reduced probability for an electron to tunnel from a pure electronic state in the lead to a polaronic state in the dot. When the temperature is increased, the competition between the thermally generated vibronic levels and the polarons gives rise to a non-monotonic behavior. This is particularly apparent for $k_{\mathrm{B}} T \lesssim \lambda^{2} \hbar \omega_{0}$, where $\lambda^{2} \hbar \omega_{0}$ is the characteristic energy of a polaron. At higher temperature $k_{\mathrm{B}} T \gg \lambda^{2} \hbar \omega_{0}$, the polaronic blockade is completely overcome and the conductance follows the Breit-Wigner scaling $1 / T$.

We have also performed numerical fits to the experimental data (Fig. 3), with $\lambda$, $\hbar \omega_{0}$, and $\gamma=\Gamma_{\mathrm{L}} \Gamma_{\mathrm{R}} /\left(\Gamma_{\mathrm{L}}+\right.$ $\left.\Gamma_{\mathrm{R}}\right)$ as fit parameters. Here, $\Gamma_{\mathrm{L} / \mathrm{R}}$ is the electronic coupling to the left/right electrical lead and $\Gamma=\Gamma_{\mathrm{L}}+\Gamma_{\mathrm{R}}$. The determined parameters are listed in the inset to Fig. 3. The large values of $\lambda=1.6-1.9$ are consistent with the Franck-Condon blockade observed at low temperature. They are also of the same order as the nanoelectromechanical coupling constants determined for suspended empty nanotubes [11].

A simple estimation of the longitudinal confining potential gives a further insight on fullerene vibrations inside the nanotube. We start from Girifalco's universal potential for graphitic structures [16], assuming interaction of a $\mathrm{C}_{60}$ molecule with its nearest-neighbor molecule on either side. The resulting potential can be approximated by a harmonic potential with a force constant 


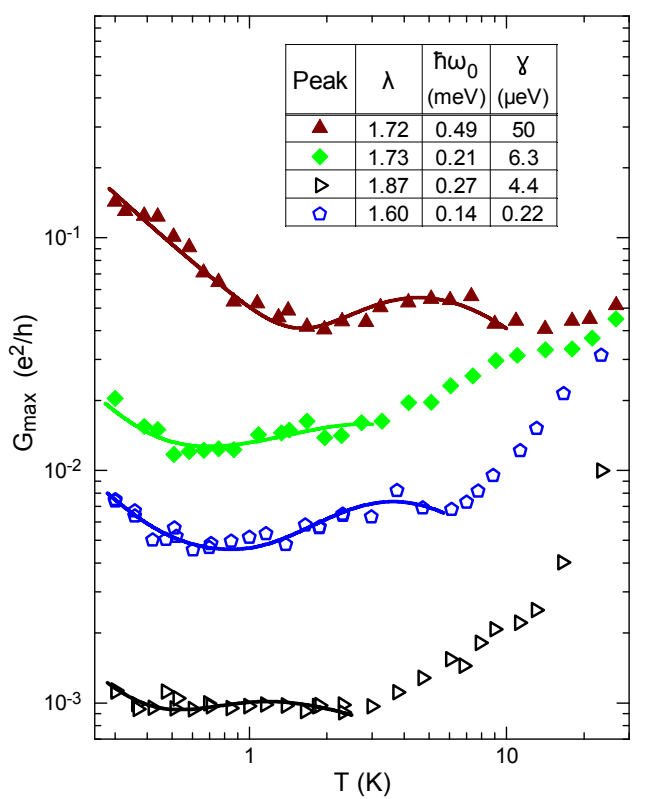

Fig. 3. $G_{\max }$ vs. $T$ for conductance peaks deviating from the Breit-Wigner model. Solid and open symbols indicate data points measured for two different peapod devices, whereas solid lines are fits to the experimental data calculated using the polaronic model described in the text. The inset gathers the determined values of the fitting parameters.

$k=2.2 \mathrm{~N} \mathrm{~m}^{-1}$. To match the experimentally determined $\hbar \omega_{0}=0.25 \mathrm{meV}$, a mass of $1.2 \times 10^{-23} \mathrm{~kg}$ (about ten times larger than that of a single $\mathrm{C}_{60}$ molecule) is thus required. This indicates that the relevant vibrations may involve clusters of about ten $\mathrm{C}_{60}$ fullerenes vibrating in phase. For densely packed peapods, such clusters could result from, e.g., local tube corrugations or defects (openings) on the nanotube's side wall.

\section{Conclusion}

We have studied the low-temperature electron transport through fullerene peapods and demonstrated the presence of a nanoelectromechanical coupling in these devices. Peapods could thus be used as novel nanoelectromechanical systems (NEMS). Apart from that, the presented experimental approach provides a method to detect in situ the presence of fullerenes (or other mobile molecules) inside an individual nanotube embedded into an electronic device.

\section{Acknowledgments}

We acknowledge financial support from the European Commission FP6, Danish DSF, British EPSRC, Swedish VR and SSF, and Korean WCU programmes.

\section{References}

[1] B.W. Smith, M. Monthioux, D.E. Luzzi, Nature 396, 323 (1998).

[2] I.V. Krive, R.I. Shekhter, M. Jonson, Low Temp. Phys. 32, 887 (2006).

[3] Y.-K. Kwon, D. Tománek, S. Iijima, Phys. Rev. Lett. 82, 1470 (1999).

[4] W.L. Yang, Z.Y. Xu, H. Wei, M. Feng, D. Suter, Phys. Rev. A 81, 032303 (2010).

[5] S. Okada, S. Saito, A. Oshiyama, Phys. Rev. Lett. 86, 3835 (2001)

[6] D.J. Hornbaker, S.-J. Kahng, S. Misra, B.W. Smith, A.T. Johnson, E.J. Mele, D.E. Luzzi, A. Yazdani, Science 295, 828 (2002).

[7] T. Okazaki, S. Okubo, T. Nakanishi, S.-K. Joung, T. Saito, M. Otani, S. Okada, S. Bandow, S. Iijima, J. Am. Chem. Soc. 130, 4122 (2008).

[8] H. Shiozawa, H. Ishii, H. Kihara, N. Sasaki, S. Nakamura, T. Yoshida, Y. Takayama, T. Miyahara, S. Suzuki, Y. Achiba, T. Kodama, M. Higashiguchi, X.Y. Chi, M. Nakatake, K. Shimada, H. Namatame, M. Taniguchi, H. Kataura, Phys. Rev. B 73, 075406 (2006).

[9] P. Utko, J. Nygård, L. Noé, M. Monthioux, Appl. Phys. Lett. 89, 233118 (2006).

[10] P. Utko, R. Ferone, I.V. Krive, R.I. Shekhter, M. Jonson, L. Noé, M. Monthioux, J. Nygård, Nat. Commun. 1, 37 (2010).

[11] R. Leturcq, C. Stampfer, K. Inderbitzin, L. Durrer C. Hierold, E. Mariani, M.G. Schultz, F. von Oppen, K. Ensslin, Nature Phys. 5, 327 (2009).

[12] C.W.J. Beenakker, Phys. Rev. B 44, 1646 (1991).

[13] M. Galperin, M.A. Ratner, A. Nitzan, J. Phys., Condens. Matter 19, 103201 (2007).

[14] I.V. Krive, R. Ferone, R.I. Shekhter, M. Jonson, P. Utko, J. Nygård, New J. Phys. 10, 043043 (2008).

[15] J. Koch, F. von Oppen, Phys. Rev. Lett. 94, 206804 (2005).

[16] L.A. Girifalco, M. Hodak, R.S. Lee, Phys. Rev. B 62, 13104 (2000). 